\title{
SAFETY AND CARDIOVASCULAR EFFICACY OF ANTI-PCSK9 MONOCLONAL ANTIBODIES: A META-ANALYSIS OF RANDOMISED CONTROLLED TRIALS
}

\section{Casula ${ }^{1,2}$, E. Olmastroni ${ }^{1}$, M.T. Boccalari ${ }^{1}$, E. Tragni ${ }^{1}$, A. Pirillo ${ }^{2,3}$, A.L. Catapano ${ }^{1,2,3}$}

1 Epidemiology and Preventive Pharmacology Service (SEFAP), Department of Pharmacological Sciences, University of Milan, Italy 2 IRCCS MultiMedica, Sesto S. Giovanni (MI), Italy

3 S.I.S.A. Center for the Study of Atherosclerosis - Bassini Hospital, Cinisello Balsamo (MI), Italy

\section{BACKGROUND}

Proprotein convertase subtilisin-kexin type 9 (PCSK9) is a serine protease crucially involved in lipid metabolism by mediating LDL receptor clearance.

Since the role of PCSK9 in LDLR degradation and LDLcholesterol metabolism was discovered, different pharmacological approaches to inhibit this protein and lower plasma LDL-C have been developed. Among the monoclonal antibodies (mAbs) developed against PCSK9, clinical trial results are available for alirocumab (SAR236553/REGN727) and evolocumab (AMG145)

Data from randomized controlled trials (RCTs) assessing the impact of PCSK9 mAbs on cardiovascular (CV) outcomes have indicated a clear clinical benefit in subjects at high CV risk.

\section{AIM}

Aim of this meta-analysis was to investigate the safety and efficacy of treatment with PCSK9 antibodies, particularly with respect to their effect on clinical outcomes, in all published RCTs, updating the available results.

\section{METHODS}

Pubmed, MEDLINE, and EMBASE were searched from inception until November 2018.

The search strategy included keywords and MeSH terms relating to the PCSK9 inhibitors evolocumab and alirocumab. The inclusion criteria were:

(1) English language;

(2) phase 2 or 3 RCTs

(3) comparing PCSK9 antibodies with placebo;

(4) reporting data on CV outcomes;

(5) treatment duration longer than 8 weeks.

Primary clinical end points were all-cause mortality and cardiovascular mortality; secondary end points were CV events (myocardial infarction, unstable angina, revascularization, heart failure, cerebrovascular events, stroke), and serious adverse events (as reported in each $\mathrm{RCT}$ ).

Odds ratios (ORs) with 95\% Cls were used as summary statistics. Between-study heterogeneity was measured with the $\mathrm{I}^{2}$ statistics.

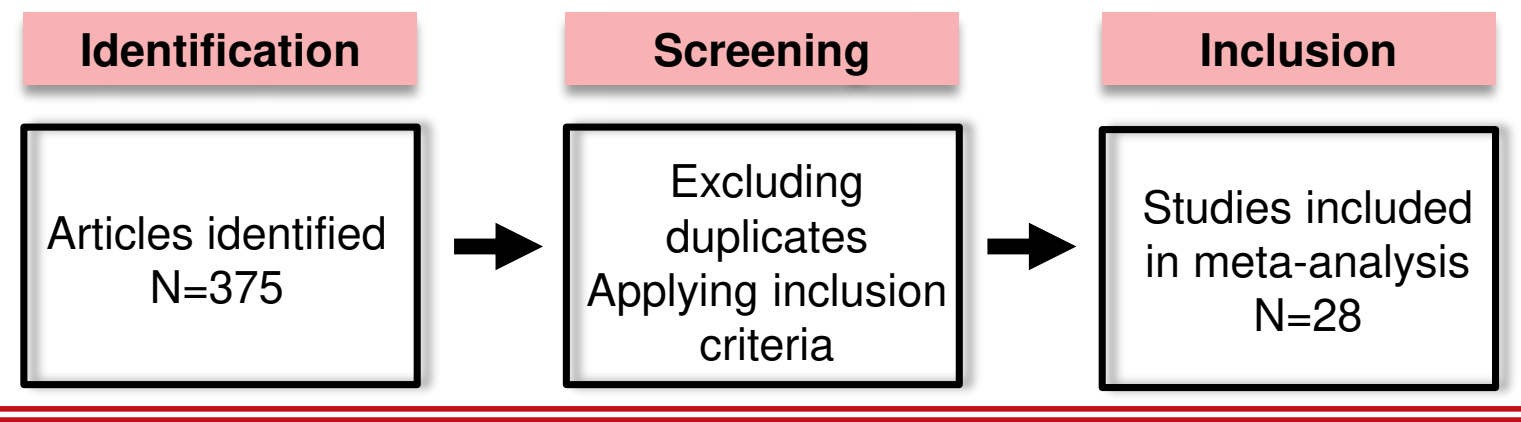

\section{CONCLUSIONS}

We observed a significant reduction in the risk of CV events, a small but significant reduction of serious adverse events, and no differences in either all-cause or cardiovascular mortality.

These results suggest that:

(1) the inhibition of PCSK9 significantly improves cardiovascular outcomes

(2) the treatment with PCSK9-mAbs appears to be safe.

Despite that, no significant cardiovascular mortality benefit seems to be associated with PCSK9 mAb treatment. It might be achievable with a longer observation period, since translation of cholesterol lowering into a cardiovascular benefit requires time.

\section{RESULTS}

The 28 selected studies included 62,281 participants $(33,204$ in the mAb arm, 29,077 in the placebo arm); all of them were RCTs comparing evolocumab or alirocumab with placebo (mainly on top of a statin). The treatment follow-up ranged from 8 weeks up to 208 weeks.
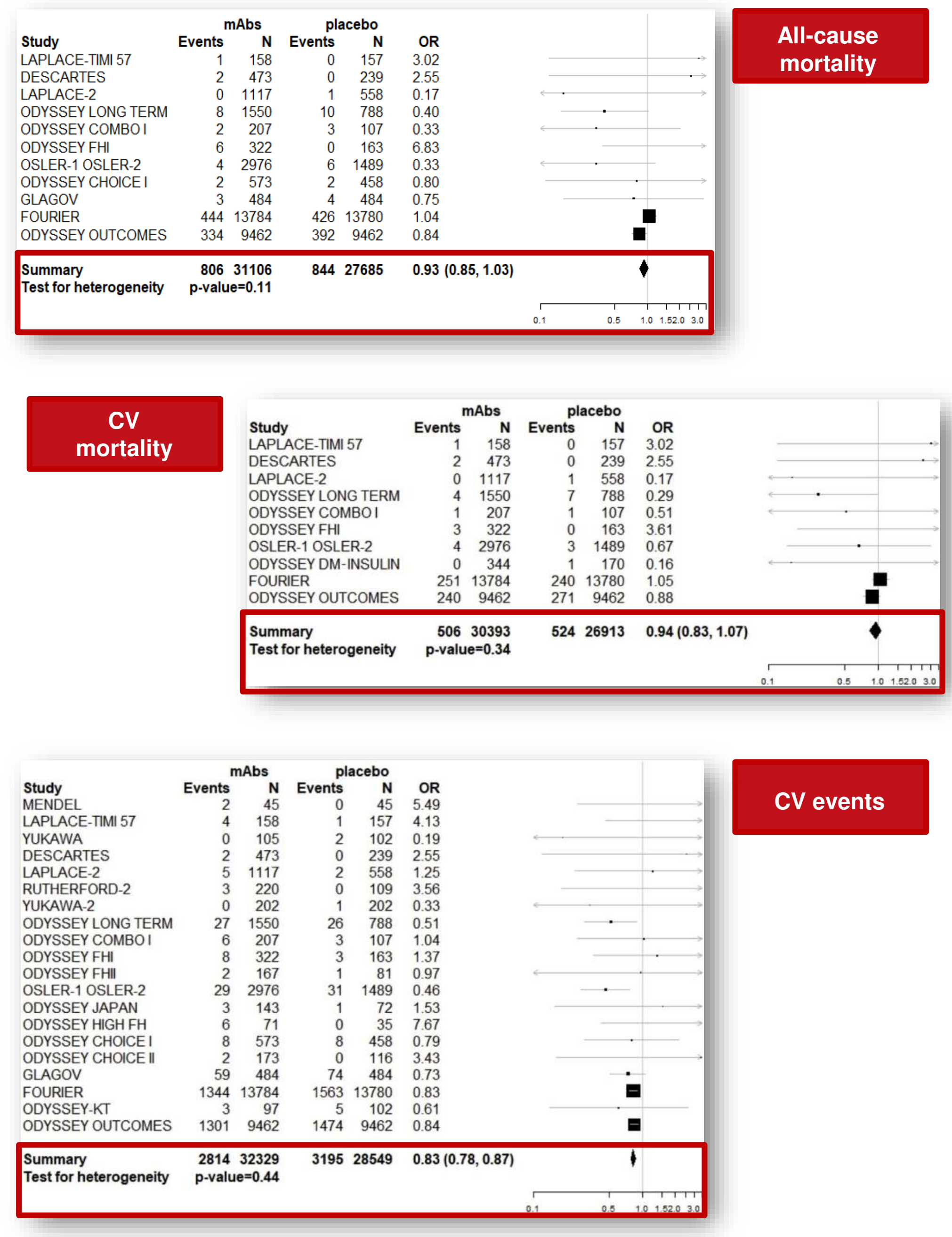

CV events

Serious adverse events

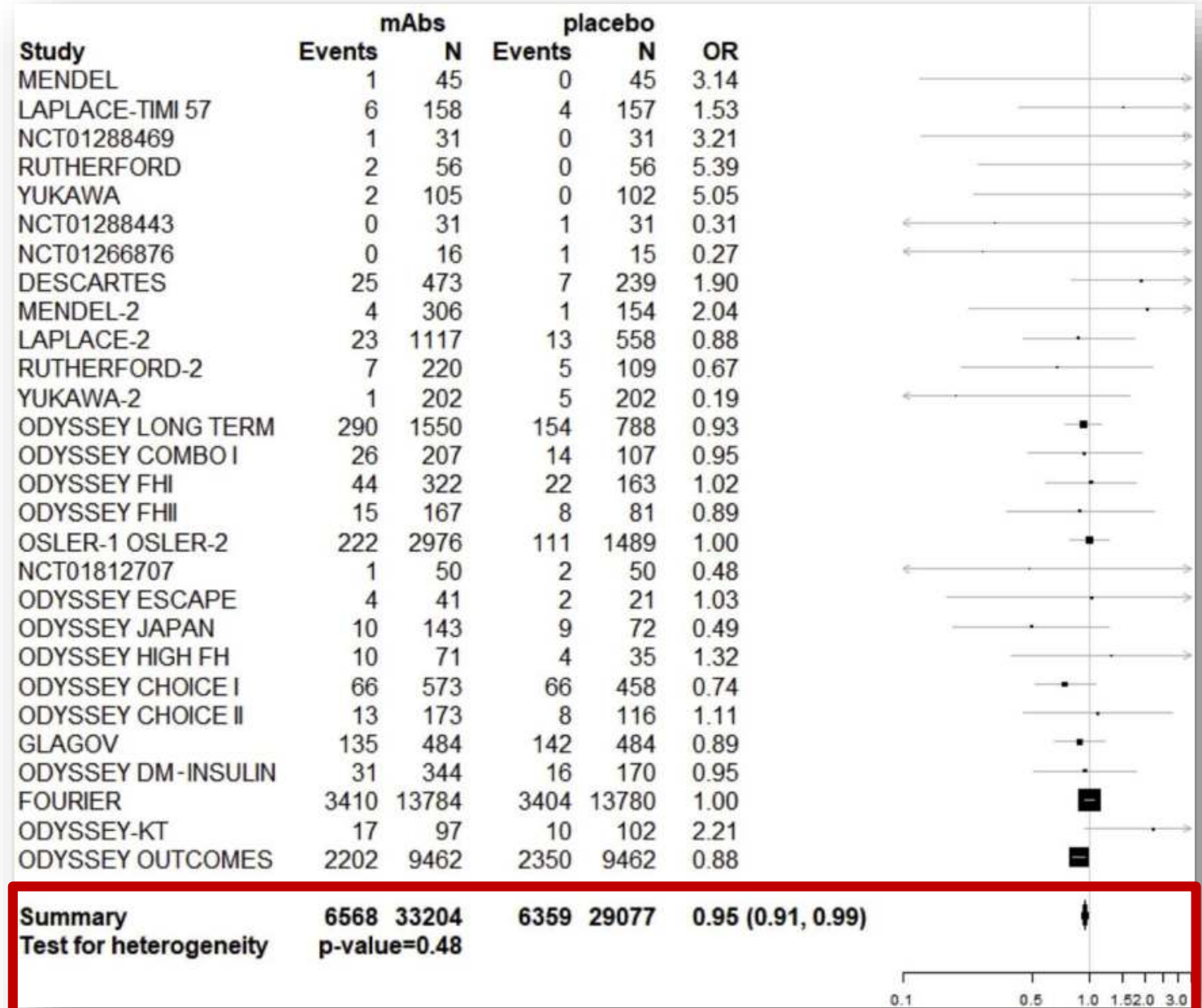

